\title{
Characterization of Intraductal Papillary Neoplasm of the Bile Duct with Respect to the Histopathologic Similarities to Pancreatic Intraductal Papillary Mucinous Neoplasm
}

\author{
Yasuni Nakanuma ${ }^{1,2}$, Yuko Kakuda ${ }^{1}$, and Katsuhiko Uesaka ${ }^{3}$ \\ ${ }^{1}$ Department of Diagnostic Pathology, Shizuoka Cancer Center, Sunto, ${ }^{2}$ Department of Diagnostic Pathology, Fukui Prefecture Saiseikai Hospital, \\ Fukui, and ${ }^{3}$ Department of Hepatobiliary Pancreatic Surgery, Shizuoka Cancer Center, Sunto, Japan
}

Intraductal papillary neoplasms of the bile duct (IPNBs) are known to show various pathologic features and biological behaviors. Recently, two categories of IPNBs have been proposed based on their histologic similarities to pancreatic intraductal papillary mucinous neoplasms (IPMNs): type 1 IPNBs, which share many features with IPMNs; and type 2 IPNBs, which are variably different from IPMNs. The four IPNB subtypes were re-evaluated with respect to these two categories. Intestinal IPNBs showing a predominantly villous growth may correspond to type 1 , while those showing papillay-tubular or papillay-villous growth correspond to type 2. Regarding gastric IPNB, those with regular foveolar structures with varying numbers of pyloric glands may correspond to type 1, while those with papillary-foveolar structures with gastric immunophenotypes and complicated structures may correspond to type 2. Pancreatobiliary IPNBs that show fine ramifying branching may be categorized as type 1 , while others containing many complicated structures may be categorized as type 2. Oncocytic type, which displays solid growth or irregular papillary structures, may correspond to type 2, while papillary configurations with pseudostratified oncocytic lining cells correspond to type 1 . Generally, type 1 IPNBs of any subtype develop in the intrahepatic bile ducts, while type 2 IPNBs develop in the extrahepatic bile duct. These findings suggest that IPNBs arising in the intrahepatic ducts are biliary counterparts of IPMNs, while those arising in the extrahepatic ducts display differences from prototypical IPMNs. The recognition of these two categories of IPNBs with reference to IPMNs and their anatomical location along the biliary tree may deepen our understanding of IPNBs. (Gut Liver 2019;13:617-627)
Key Words: Intraductal papillary neoplasm of bile duct; Cholangiocarcinoma; Intraductal papillary mucinous neoplasm of pancreas; Biliary anatomy; Counterpart

\section{INTRODUCTION}

Intraductal papillary neoplasms of the bile duct (IPNBs) are characterized by dilated bile ducts that are filled with preinvasive papillary or villous biliary neoplasms covering fine fibrovascular stalks and a lack of an ovarian-type stroma (Fig. $1 \mathrm{~A}$ and $\mathrm{B}$ ) and are regarded as preinvasive biliary lesions that are eventually followed by stromal invasion (IPNB associated with an invasive carcinoma).$^{1-5}$ In addition, IPNBs show a variable intramucosal spread of neoplastic epithelia around the main papillary tumor (Fig. 1C); in some cases, this intramucosal spread is extensive. Intraductal papillary mucinous neoplasms (IPMNs) of the pancreas are also a preinvasive intraductal papillary neoplasm and are characterized by the intramucosal spread of neoplastic epithelia followed by invasive carcinoma. ${ }^{1,3,6} \mathrm{Al}-$ most all IPMN cases are associated with excessive intraductal mucin hypersecretion and show different clinicopathological features and genetic molecular alterations according to their anatomical locations (main pancreatic duct and branch duct) and the four subtypes into which they are classified (intestinal, gastric, pancreatobiliary [PB], and oncocytic subtype). ${ }^{7}$

IPNBs have been the topic of many recent studies, including studies on their clinicopathological features and imaging findings, and it was found that IPNBs show variable gross and histopathological features and biological behaviors. ${ }^{3,8-18}$ It is noteworthy that the incidence of stromal invasion and the histologic features differ among several of the proposed subcategories of IPNB. $^{9,12,17,19}$ There have been several studies on the histological

\footnotetext{
Correspondence to: Yasuni Nakanuma

Department of Diagnostic Pathology, Fukui Prefecture Saiseikai Hospital, Wadanakacho Funahashi 7-1, Fukui 918-8503, Japan

Tel: +81-776-28-8527, Fax: +81-776-23-1111, E-mail: nakanuma@staff.kanazawa-u.ac.jp

Received on October 19, 2018. Revised on December 2, 2018. Accepted on December 3, 2018. Published online April $15,2019$. pISSN 1976-2283 eISSN 2005-1212 https://doi.org/10.5009/gnl18476

@ This is an Open Access article distributed under the terms of the Creative Commons Attribution Non-Commercial License (http://creativecommons.org/licenses/by-nc/4.0) which permits unrestricted non-commercial use, distribution, and reproduction in any medium, provided the original work is properly cited.
} 


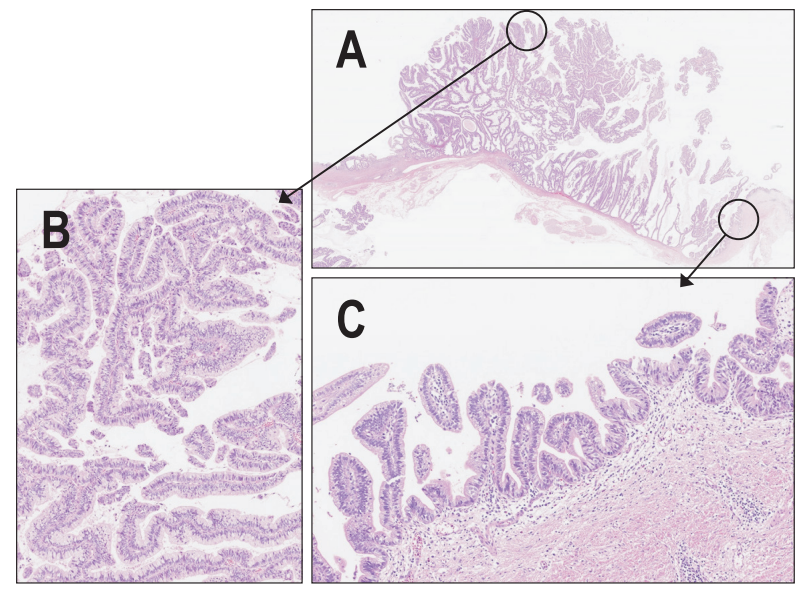

Fig. 1. Intraductal papillary neoplasm of the bile duct arising in the extrahepatic bile duct. (A) A papillary lesion and laterally spreading lesion in the extrahepatic bile duct (H\&E, x50). (B) A higher-magnification view of the papillary lesions marked by circle in A shows fine fibrovascular cores covered by neoplastic epithelia (H\&E, x70). (C) A higher-magnification view of the laterally spreading lesion marked by circle in A shows micropapillary and flat neoplastic epithelia (H\&E, $\times 100$ ).
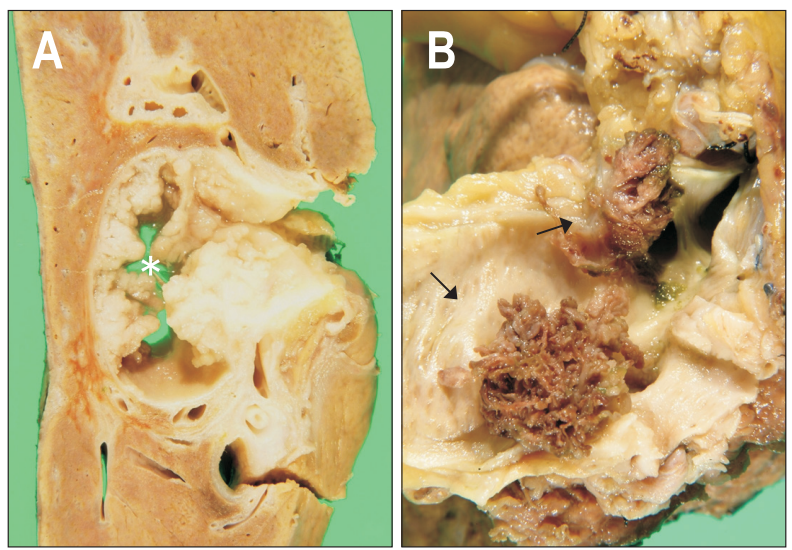

Fig. 2. The gross features of an intraductal papillary neoplasm of the bile duct (IPNB). (A) An IPNB growing in the intrahepatic bile duct showing cystic dilatation (white asterisk, dilated bile duct lumen). (B) IPNB in the extrahepatic bile duct showing villous features (one tumor was separated into two [arrows] at the time of dissection).

similarities between IPNBs and IPMNs. For example, IPNBs are also divided into four subtypes, ${ }^{1,3,6}$ and IPNBs also frequently show excessive intraductal mucin hypersecretion. Recently, IPNBs were also reported to present different gross and histologic features according to their anatomical location along the biliary tree, suggesting that IPNB may be a heterogeneous and complex biliary disease. ${ }^{17-20}$ This heterogeneity and complexity lead to controversies regarding the clinicopathological recognition and diagnosis of IPNB. ${ }^{4,10,21,22}$

The re-evaluation of the pathological features of IPNBs with reference to IPMNs and their anatomical location along the biliary tree may improve our understanding and recognition of the

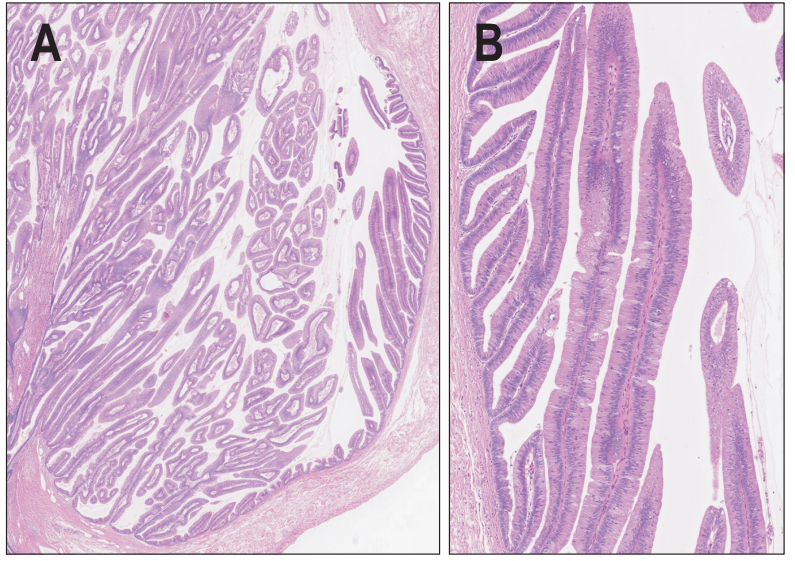

Fig. 3. Intestinal-type intraductal papillary neoplasm of the bile duct (IPNB) (type 1). (A) An IPNB arising in the intrahepatic bile duct shows a villous architecture with thin fibrovascular cores (H\&E, $\times 80)$. (B) A higher-magnification view of the villous neoplasm shows stratified nuclei with amphiphilic cytoplasm and little supranuclear mucin (H\&E, $\times 120)$.

clinicopathological aspects of IPNB and build a better consensus regarding these neoplasms.

In this review, we assessed the pathologic heterogeneity and variability of IPNBs and introduce two categories of IPNBs (type 1 and 2) with reference to their similarity to IPMN. ${ }^{22}$ We then re-evaluated the four subtypes of IPNBs based on these two categories and on their anatomical location along the biliary tree. We also reviewed several disease entities that mimic IPNB. ${ }^{4,23-27}$ In our study, the extrahepatic bile duct included the right and left hepatic ducts, while the intrahepatic bile ducts were proximal to the right and left hepatic ducts. The study protocol of this review was approved by the Institutional Review Boards of Fukui Saiseikai Hospital (2017-093) and Shizuoka Cancer Center (T286). The informed consent was waived.

\section{HETEROGENEITY IN THE PATHOLOGY OF IPNB}

Macroscopically, intrahepatic IPNBs are more likely to show unilocular or multilocular cystic dilatation of the affected ducts where papillary neoplasms are identifiable (Fig. 2A), while extrahepatic IPNBs presenting papillary or polypoid neoplasms are typically associated with cylindrical or fusiform dilatation of the affected bile ducts (Fig. 2B). ${ }^{1-3,8}$ Gross changes in extrahepatic IPNBs can also be encountered in intrahepatic IPNBs. Excessive mucin hypersecretion is more frequently observed in intrahepatic IPNBs than in extrahepatic IPNBs. ${ }^{5,28}$ Bile ducts with excessive mucin secretion located upstream and downstream from IPNBs are significantly dilated due to the large amount of mucin in the duct lumen. The anatomical location of IPNBs within or outside the liver parenchyma and the presence or absence of excessive mucin hypersecretion from an IPNB may influence the gross appearance of the IPNB. ${ }^{14,18}$

IPNBs are histologically classifiable into four subtypes based 
on the lining epithelial cells and architectures, including fibrovascular stroma: intestinal (Figs 3, 4), gastric (Figs 5, 6), PB (Figs 7, 8) and oncocytic (Figs 9, 10). While many cases are predominantly composed of an individual subtype, mixtures of other subtypes are not infrequently observed, as is the case in IPMN. ${ }^{1,2,7,7,8}$ This subtyping is facilitated by immunohistochemistry to detect mucus core proteins and cytokeratins. ${ }^{1,5}$ A recent study showed that the incidence of these subtypes differed between intrahepatic and extrahepatic IPNBs. ${ }^{5}$ As for the histological grades of intraepithelial neoplasms (dysplasia), many IPNB cases arising in the intrahepatic bile ducts are graded as highgrade dysplasia, while the areas of low-/intermediate-grade dysplasia infrequently co-exist within the same tumor. Some IPNBs are composed of only low-/intermediate-grade dysplasia. In contrast, extrahepatic IPNBs are always composed of high-
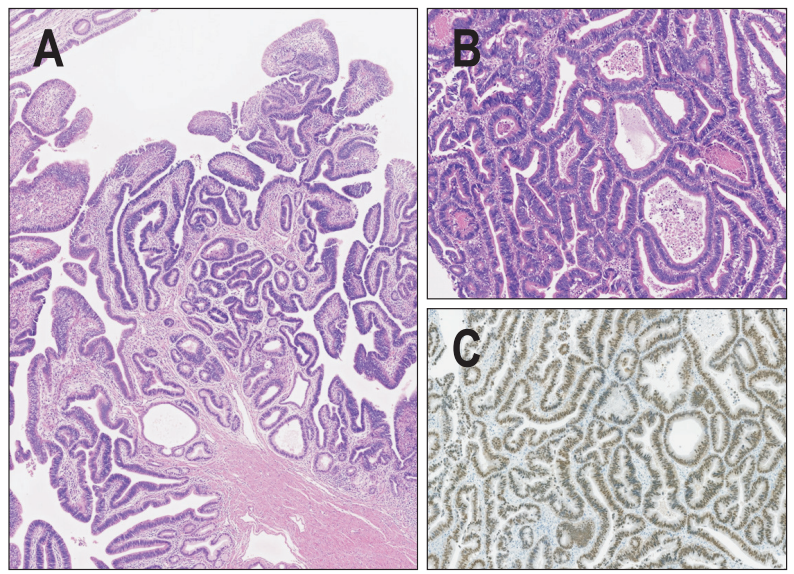

Fig. 4. Intestinal-type intraductal papillary neoplasm of the bile duct (IPNB) (type 2). (A) An IPNB arising in the distal bile duct shows papillary-tubular architecture with thin, focally fibrotic, fibrovascular cores (H\&E, x60). (B) An IPNB arising in the distal bile duct with high-grade dysplasia. The structures of the papillary-tubular patterns are irregular (H\&E, $\times 80)$. (C) These neoplastic cells are diffusely positive for CDX2 (immunostaining, $\times 70$ ).

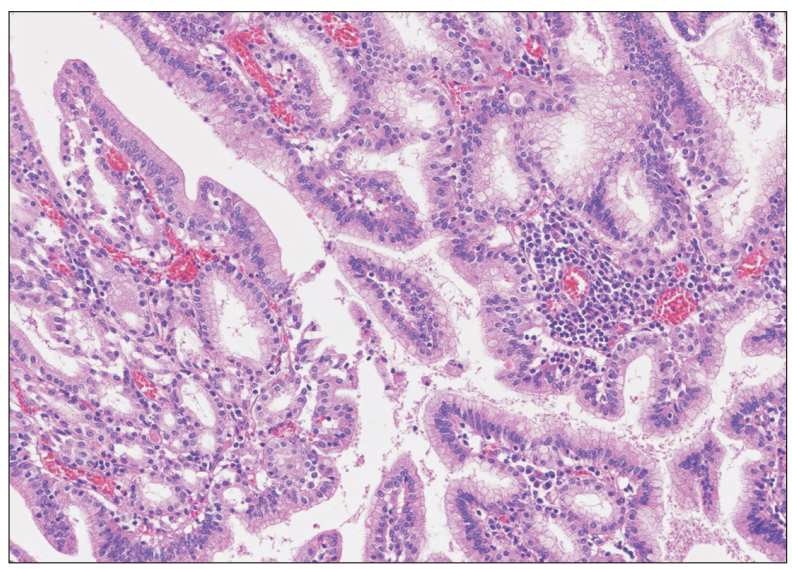

Fig. 5. Gastric-type intraductal papillary neoplasm of the intrahepatic bile duct (type 1). Foveolar epithelia and pyloric glands comprise the basic architecture of this gastric neoplasm (H\&E, $\times 150)$. grade dysplasia and in many cases are only composed of highgrade components. IPNBs, particularly those containing variable foci of low-/intermediate-grade dysplasia, may be undergoing multi-step carcinogenesis. ${ }^{1,29}$

Furthermore, unlike intrahepatic IPNBs, which are invasive at the time of resection in approximately 50\% of cases, many extrahepatic IPNBs show at least focal stromal invasion, which implies that intrahepatic IPNBs are less aggressive than extrahepatic IPNBs. In addition to the papillary and/or villous structures, other features, such as crowded tubular, cribriform or

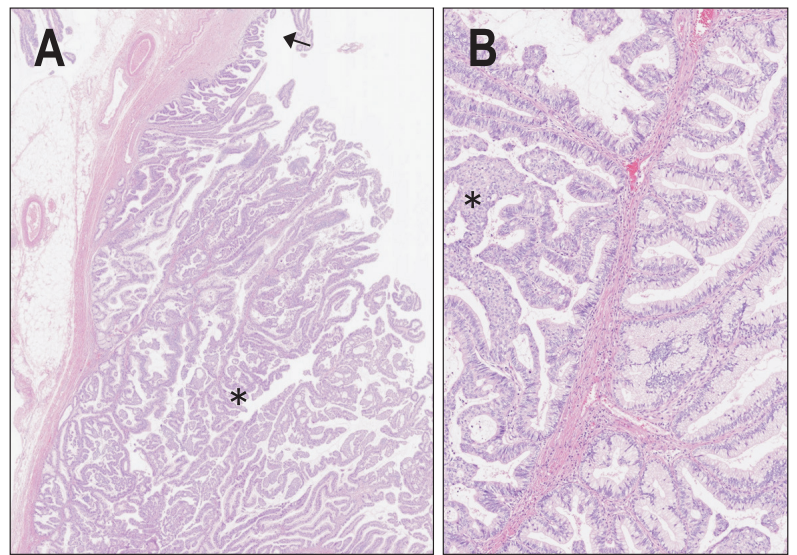

Fig. 6. Gastric-type intraductal papillary neoplasm of the extrahepatic bile duct (type 2). (A) A papillary neoplasm with fine fibrovascular cores and epithelial lining (asterisk), and flat and nonpapillary neoplastic epithelial lining in the adjacent mucosa (arrow) are identifiable (H\&E, $\times 80)$. (B) A higher-magnification view of (A) shows a low columnar clear cytoplasm and basally located nuclei appearing as pyloric glands (right half) and low columnar epithelia with less mucinous cytoplasm, reminiscent of gastric foveola (left half). Asterisk indicates solid structures $(\mathrm{H} \& \mathrm{E}, \times 120)$.
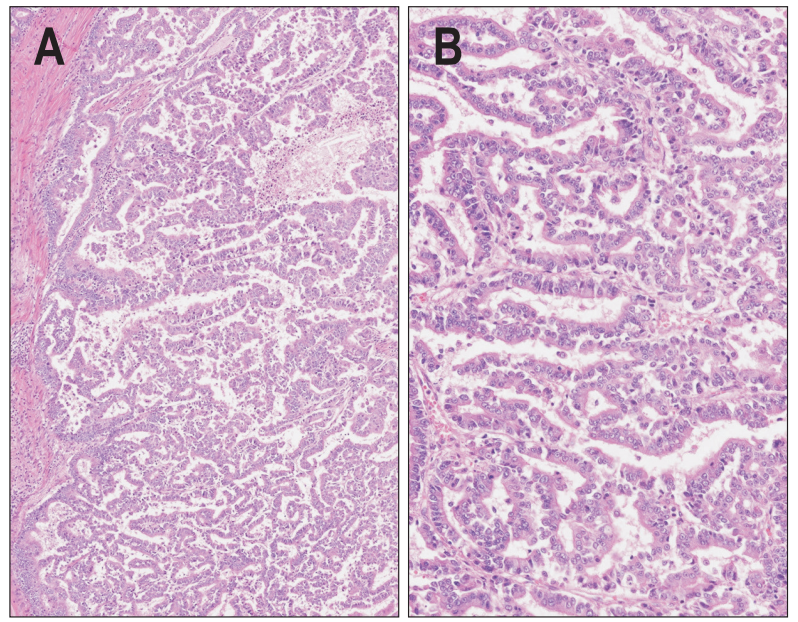

Fig. 7. Pancreatobiliary-type intraductal papillary neoplasm of the bile duct (IPNB) (type 1). (A) An IPNB arising in the intrahepatic bile duct shows fine and branching fibrovascular cores lined by columnar epithelia. (H\&E, $\times 120)$. (B) Fine and branching stalks are lined by low columnar and cuboidal epithelia. A higher-magnification view of (A) (H\&E, $\times 140)$. 


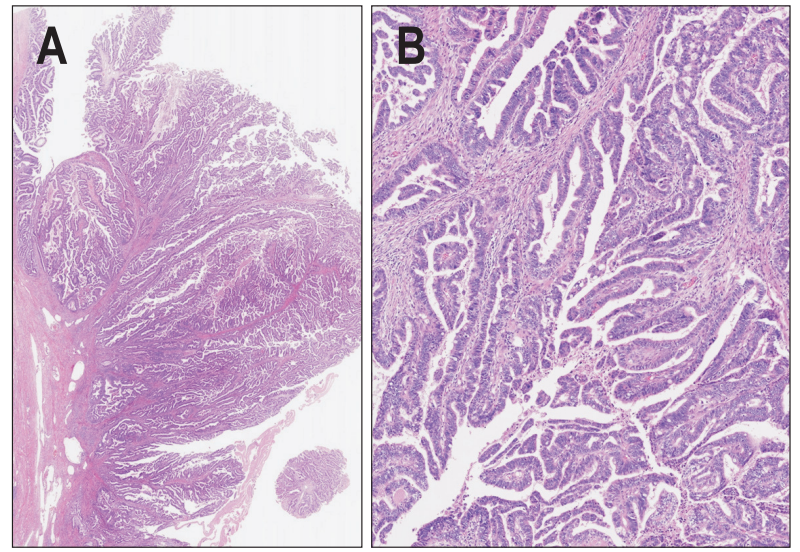

Fig. 8. Pancreatobiliary-type intraductal papillary neoplasm of the bile duct (IPNB) (type 2). (A) An IPNB arising in the extrahepatic bile duct showing fine and branching fibrovascular stalks lined by papillary neoplastic epithelia $(\mathrm{H} \& \mathrm{E}, \times 50)$. (B) The epithelial lining shows branching stalks with papillary, pseudopapillary and serrated epithelia with high-grade dysplasia $(\mathrm{H} \& \mathrm{E}, \times 120)$.
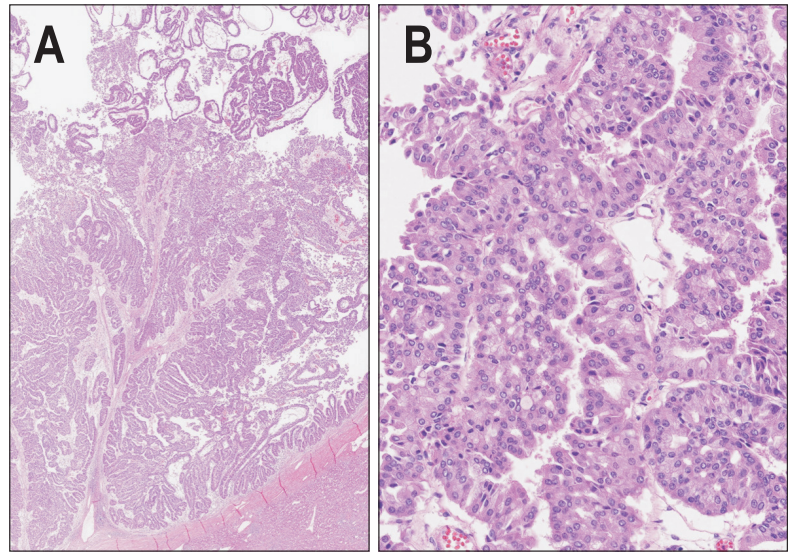

Fig. 9. Oncocytic-type intraductal papillary neoplasm of the intrahepatic bile duct (type 1). (A) A papillary neoplasm with fine fibrovascular cores and epithelial lining (H\&E, ×50). (B) Lining the epithelia are several cell layers with numerous secondary lumina and acidophilic or oncocytic cytoplasm as well as hyperchromatic nuclei. A higher-magnification view of (A) (H\&E, ×120).

solid growth patterns, may complicate the architecture of IPNBs, particularly in those of the extrahepatic bile ducts. ${ }^{22}$ Generally, papillary or villous growth with fibrovascular stalks ( $>5 \mathrm{~mm}$ in height from the adjacent biliary mucosa) are typical; however, some papillary neoplasms with a similar histopathology that are $<5 \mathrm{~mm}$ but $>3 \mathrm{~mm}$ in height are occasionally encountered. ${ }^{1,5,16,24}$ IPNBs located in the intrahepatic bile ducts tend to be larger in both mass and length than those in the extrahepatic bile ducts. ${ }^{15}$ Taken together, these findings suggest that the gross and microscopic morphologies, pathogenesis and biological activities of IPNBs may be related to their anatomical location along the biliary tree., 5,26

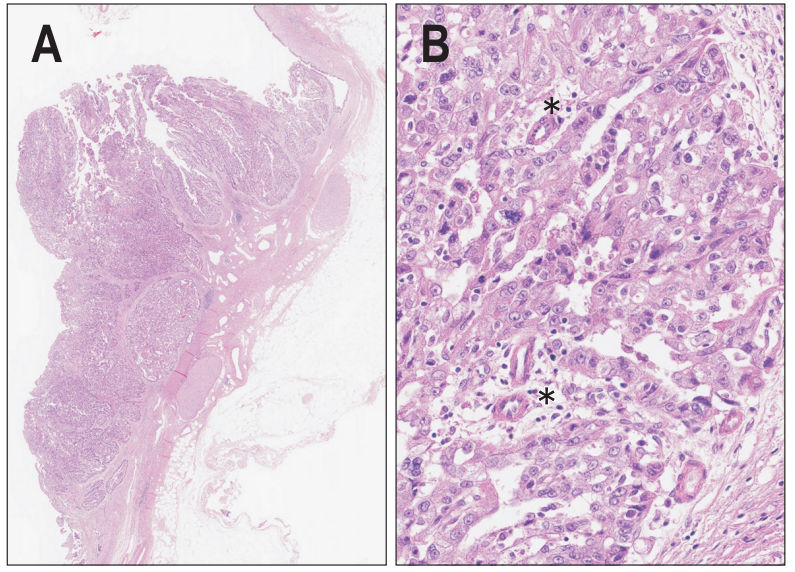

Fig. 10. Oncocytic-type intraductal papillary neoplasm of the extrahepatic bile duct (type 2). (A) A papillary neoplasm shows polypoid growth with fine fibrovascular cores and multilayered nuclei $(\mathrm{H} \& \mathrm{E}$, $\times 40$ ). (B) The papillary or compact growth of oncocytic cells with hyperchromatic and pleomorphic nuclei. The secondary lumina are vague (asterisks, edematous stalk). A higher-magnification view of (A) (H\&E, x150).

\section{SIMILARITIES BETWEEN IPNB AND PANCREATIC IPMN}

There are several biliary and pancreatic diseases that share many clinical and pathological features ${ }^{5,15,16,18,25,26,30,31}$ and can be regarded as a spectrum of "biliary diseases with pancreatic counterparts." The representative diseases are shown in Table 1. The pathological and clinical similarities between IPNB and IPMN have been reported, ${ }^{5,15,18,22,30}$ suggesting that IPNBs and IPMNs can be included in this spectrum.

There are many anatomical, embryological and experimental data or background characteristics that support the occurrence of similar diseases in the biliary tract and pancreas; $;^{30,32-36}$ some of these are shown in Table 2. They suggest that the bile duct is inherently accompanied by variable pancreatic features, making it plausible for diseases with similar features to develop in the pancreas as well as the biliary tract. ${ }^{27,30}$

\section{The similarities and differences between IPNB and IPMN}

IPNBs and IPMNs may present with similar histological features and biological behaviors and may undergo similar pathogenetic processes. However, the bile ducts and pancreas themselves are different organs, so it is conceivable that IPNB and IPMN are not completely identical. According to our recent studies, approximately half of IPNBs showed similar histopathological features to IPMNs, while the other half did not. ${ }^{5,15}$ The similarities and differences between IPNBs and IPMNs are summarized in Table 3.

IPMNs can involve the main pancreatic duct (main duct type), branch duct (branch duct type), or both ducts (combined type). The branch duct type of IPMN is usually multicystic and of the gastric subtype. While IPNBs involving the extrahepatic bile ducts and intrahepatic large bile duct may correspond to 
Table 1. Nonneoplastic and Neoplastic Biliary and Pancreatic Diseases Sharing Common Pathologic Features (Biliary Diseases with Pancreatic Counterparts)

Nonneoplastic diseases

- IgG4-related sclerosing cholangitis and type I autoimmune pancreatitis: inflammatory diseases with similar features in the bile duct and pancreas and belonging to the family of systematic IgG4-related disease. Almost always found in the same patients.

- Follicular cholangitis and follicular pancreatitis: show similar features in the bile duct and pancreas.

- Cystic fibrosis of the bile duct and pancreatic duct: same mechanisms involved.

Neoplastic diseases

- Mucinous cystic neoplasm of the hepatobiliary system and pancreas: present the same clinical and histologic features.

- Perihilar and distal cholangiocarcinoma (p/dCCA) and pancreatic duct and adenocarcinoma (PDAC): show similar pathologic features and biological behaviors.

- Biliary intraepithelial neoplasm (BilIN) and pancreatic intraepithelial neoplasm (PanIN): BilINs develop in the bile duct as the precursor of p/dCCA while PanINs develop in the pancreatic duct as the precursor of PDAC. Both share many histopathological and molecular features.

- Intraductal tubulopapillary neoplasia of the bile duct and pancreas: share many pathologic features.

- Intraductal papillary neoplasm of bile duct and intraductal papillary mucinous neoplasm of pancreas: share many features.

Table 2. Anatomical and Embryological Backgrounds for the Occurrence of Several Diseases in the Bile Duct and Pancreas Sharing Many Pathologic Features

Anatomic and embryologic factors

- The biliary tract and pancreas are closely located anatomically and function cooperatively. They share several developmental processes and are derived from the endoderm of the foregut at almost the same time. In addition, the same several transcription factors, such as HES1 and PDX2, are involved in their development.

- The ventral pancreas, which is derived from the extrahepatic bile duct, revolves eventually to unite the dorsal pancreas and form the pancreas. The peribiliary glands physiologically located around the biliary tract may be reminiscent of the ventral pancreas along the biliary tree for the following reasons:

1) Small amounts of pancreatic exocrine acini are intermingled with these glands.

2) Pancreatic exocrine enzymes are detected in the peribiliary glands.

3) Peribiliary glands drain into the bile duct lumen.

- Stem cells or progenitor cells for cholangiocytes and pancreatic cells are physiologically located in the peribiliary glands and are involved in the regeneration and pathology of the bile duct.

Experimental animal studies

- HES1-deficient mice: the biliary tract is replaced by pancreatic differentiation, suggesting the potential conversion of the biliary tract to the pancreas under pathologic conditions.

the main pancreatic duct type, the counterparts of branch-type IPMN in the biliary tract remain speculative. Cystic micropapillary neoplasms in the peribiliary glands, which usually show gastric immunophenotypes, ${ }^{16,37,38}$ may be counterparts of branch-type IPMN.

Four subtypes are recognizable in IPNB and IPMN, ${ }^{1,2,7}$ although the histology of subtypes of IPNBs and IPMNs are not the same in all cases, and morphologic variations of IPNBs remain to be more characterized (see below). The excessive mucin hypersecretion from the neoplastic epithelium into the ductal lumen is highly characteristic of IPMN. Thus, IPNB with excessive mucin hypersecretion may be a real counterpart of IPMN, while IPNB without mucin hypersecretion may be another category of IPNB. ${ }^{14,17,19}$ Both IPNBs and IPMNs show various grades of dysplasia, ranging from low-/intermediate- to high-grade dysplasia. IPNBs predominantly show high-grade dysplasia, and main pancreatic duct-type IPMNs are also often high-grade, although the gastric subtype of the branch duct type of IPMNs usually shows low-/intermediate-grade dysplasia. Interestingly, many IPNBs show papillary or villous structures that are shared by pancreatic IPMNs microscopically, while some IPNBs harbor more complicated architecture with foci that show a solid and cribriform or crowded tubular growth in addition to the papillary or villous architecture. ${ }^{16,22}$

IPNB and IPMN are eventually followed by an invasive adenocarcinoma. Extensive sampling may be required to identify small foci of invasion, particularly in tumors with an extensive flat or nonpapillary neoplastic spread around the papillary tumors. ${ }^{7,39}$ Invasive parts of IPNB usually show tubular adenocarcinoma with desmoplastic reaction and only occasionally 
Table 3. Similarities and Differences in the Pathologic Features and Architecture of IPNB and IPMN of the Pancreas

\begin{tabular}{|c|c|c|}
\hline & Similarities & Differences \\
\hline $\begin{array}{l}\text { Gross and microscopic } \\
\text { lesions }\end{array}$ & $\begin{array}{l}\text { 1) IPNB and IPMN are preinvasive papillary neoplastic le- } \\
\text { sions. } \\
\text { 2) Flat or less-papillary intraepithelial neoplasms are } \\
\text { frequently found around the main papillary neoplasm in } \\
\text { IPNB and IPMN. }\end{array}$ & $\begin{array}{l}\text { 1) IPMNs show cystic or multicystic dilatation of the } \\
\text { affected ducts. Intrahepatic IPNBs show cystic or mul- } \\
\text { ticystic changes, whereas extrahepatic IPNBs } \\
\text { show cylindrical or fusiform dilatation } \\
\text { 2) IPNBs are usually larger in both mass and length than } \\
\text { IPMN. }\end{array}$ \\
\hline Four subtypes & Four subtypes are recognizable in IPNB and also IPMN. & $\begin{array}{l}\text { The histologies of these subtypes are not exactly the } \\
\text { same between IPNB and IPMN. }\end{array}$ \\
\hline $\begin{array}{l}\text { Excessive mucin } \\
\text { hypersecretion into } \\
\text { the ductal lumen }\end{array}$ & $\begin{array}{l}\text { Excessive mucin hypersecretion is almost always found in } \\
\text { IPMN and frequently found in intrahepatic IPNB. }\end{array}$ & $\begin{array}{l}\text { Excessive mucin hypersecretion is uncommon in IPNBs } \\
\text { in the extrahepatic bile duct. }\end{array}$ \\
\hline $\begin{array}{l}\text { Grades of intraepithelial } \\
\text { neoplasia of papillary } \\
\text { lesions }\end{array}$ & $\begin{array}{l}\text { IPNB and IPMN show various grades of dysplasia ranging } \\
\text { from low/intermediate to high-grade dysplasia. }\end{array}$ & $\begin{array}{l}\text { 1) Most IPNBs show high-grade dysplasia. } \\
\text { 2) The main pancreatic duct type of IPMN is high grade. } \\
\text { The branch duct gastric subtype generally shows low/ } \\
\text { intermediate grade dysplasia. }\end{array}$ \\
\hline $\begin{array}{l}\text { Complicated } \\
\text { architectures }\end{array}$ & $\begin{array}{l}\text { Some IPNBs show similar or nearly identical papillary or } \\
\text { villous architectures to IPMNs both phenotypically and } \\
\text { architecturally. }\end{array}$ & $\begin{array}{l}\text { Some IPNBs show complicated architectures with foci of } \\
\text { solid and cribriform or tubular growth in addition to } \\
\text { papillary structures. These features are usually absent } \\
\text { in IPMNs. }\end{array}$ \\
\hline Stromal invasion & $\begin{array}{l}\text { 1) IPNB and IPMN are eventually followed by an invasive } \\
\text { adenocarcinoma at the base of the papillary lesion as } \\
\text { well as at the surrounding flat intraepithelial neoplastic } \\
\text { lesions. } \\
\text { 2) Intrahepatic IPNBs infrequently show invasion while } \\
\text { extrahepatic IPNBs frequently show invasion. IPMN of } \\
\text { main duct type frequently shows invasion, while branch } \\
\text { type IPMN infrequently shows invasion. }\end{array}$ & $\begin{array}{l}\text { 1) In invasive parts of IPMN, colloid carcinoma is a } \\
\text { frequent feature, and tubular or oncocytic carcinomas } \\
\text { with desmoplastic reaction are infrequently found. } \\
\text { 2) In invasive areas of IPNB, tubular adenocarcinoma } \\
\text { with desmoplastic reaction is found in almost all cases, } \\
\text { and colloid or oncocytic carcinoma is occasionally } \\
\text { found. }\end{array}$ \\
\hline
\end{tabular}

IPNB, intraductal papillary neoplasm of the bile duct; IPNB, intraductal papillary mucinous neoplasm.

Table 4. Two Categories of Intraductal Papillary Neoplasm of the Bile Duct

\begin{tabular}{|c|c|c|}
\hline & Type 1 & Type 2 \\
\hline $\begin{array}{l}\text { Relation to prototypes of IPMN } \\
\text { (individual subtypes) }\end{array}$ & Similar to prototypes of IPMN & Variably different from prototypes of IPMN \\
\hline Regularity of architecture & Regular and homogeneous & Irregular and variable \\
\hline Solid, cribriform, crowded tubular patterns & Rare & $\begin{array}{l}\text { Variably present, in addition to papillary } \\
\text { or villous patterns }\end{array}$ \\
\hline $\begin{array}{l}\text { Cellular and nuclear pleomorphism } \\
\text { and atypia }\end{array}$ & Mild atypia & $\begin{array}{l}\text { Pleomorphic and increased atypia } \\
\text { (including overt malignant changes) }\end{array}$ \\
\hline Excessive mucin hypersecretion & Frequent & Uncommon \\
\hline Stromal invasion & Less than $50 \%$ & More than 80\% \\
\hline Anatomical location along the biliary tree & $\begin{array}{l}\text { Proximal intrahepatic branches to right } \\
\text { or left hepatic ducts }\end{array}$ & $\begin{array}{l}\text { Extrahepatic bile duct, including the right } \\
\text { and left hepatic ducts }\end{array}$ \\
\hline
\end{tabular}

IPMN, intraductal papillary mucinous neoplasm. 
show oncocytic or colloid carcinoma, while the invasive parts of IPMNs are frequently associated with colloid carcinoma with or without tubular adenocarcinoma components. ${ }^{7}$

\section{The subclassification of IPNBs based on their similarities to IPMNs}

Based on the above-mentioned similarities and differences between IPNBs and IPMNs and on the presence or absence of complicated lesion(s), IPNBs can be subdivided into two categories: types 1 and 2 (Table 4). ${ }^{16}$ Recently, a Japan-Korea study group on IPNBs, which was jointly sponsored by the Japan Biliary Association and the Korean Association of Hepato-Biliary and Pancreas Surgery, supported the classification of IPNBs into these two categories. ${ }^{22}$

\section{1) Type 1 IPNB}

This type (Figs 3, 5, 7, 9) resembles IPMN histologically and is usually found in the intrahepatic bile duct. Growth patterns are relatively uniform or regular within individual tumors. Papillary fibrovascular stalks are generally thin, but occasional foci of edematous broad stroma may also be present. In addition to the papillary or villous architecture, tubular components may co-exist, as they do in gastric-type IPMN (pyloric gland components), and they are relatively well organized. Gross mucin in the dilated duct is common (found in roughly 80\% of cases). While many IPNBs of this type show high-grade dysplasia, some contain foci of low-/intermediate-grade dysplasia while others are completely composed of low-/intermediate-grade dysplasia. This type is associated with invasive carcinomas in less than $50 \%$ of cases at the time of surgical resection. ${ }^{22}$

\section{2) Type 2 IPNB}

Generally, this type (Figs 4, 6, 8, 10) shows various differences from prototypical IPMNs and typically develops in the extrahepatic bile duct. The papillary architecture is mainly composed of thin fibrovascular stalks, although such stalks are variably widened at the basal side in some cases and are often associated with irregular or complicated branching or papillary architecture. Crowded tubular, cribriform and solid components may also be observed within the tumor. Grossly identifiable mucin overproduction is uncommon. The noninvasive components show predominantly high-grade dysplasia (in all cases) with an occasional identifiable component of low-/intermediate-grade dysplasia. Many cases are associated with invasive adenocarcinoma.

\section{The anatomical location of the two categories of IPNB along the biliary tree}

Why some IPNB cases show histological similarities to IPMN and others do not is unclear at present. Type 1 is frequently found in the intrahepatic bile duct, while type 2 is frequently found in the extrahepatic bile duct, including the hilar bile ducts, suggesting that the anatomical locations of IPNB along the biliary tree are important in the development of the two categories. Embryologically, the origin and development of the bile ducts are thought to differ, with the bile ducts proximal to the right and left bile ducts derived from the ductal plates and remodeled bile ducts around the hepatic parenchyma, which are derived from albumin-positive hepatoblasts in the hepatic diverticulum, and the bile ducts distal from the extrahepatic bile ducts derived from the albumin-negative hepatoblasts in the hepatic diverticulum (Fig. 11)..$^{33,34,40}$ These embryological and anatomic differences in the biliary tree may be related to the development of the two categories of IPNB.

However, some IPNB cases may have overlapping features of type 1 and 2; thus, classifying such IPNBs into either of the two types is difficult, and some cases show type 1 and 2 IPNBs synchronously and/or asynchronously in the intrahepatic and extrahepatic bile ducts, suggesting that type 1 and 2 IPNBs are part of a spectrum of IPNBs along the biliary tree.

\section{THE RE-EVALUATION OF THE FOUR SUBTYPES OF IPNB WITH REFERENCE TO THE TWO CATEGORIES (TYPES 1 AND 2)}

In our experience, the histological features characterizing the

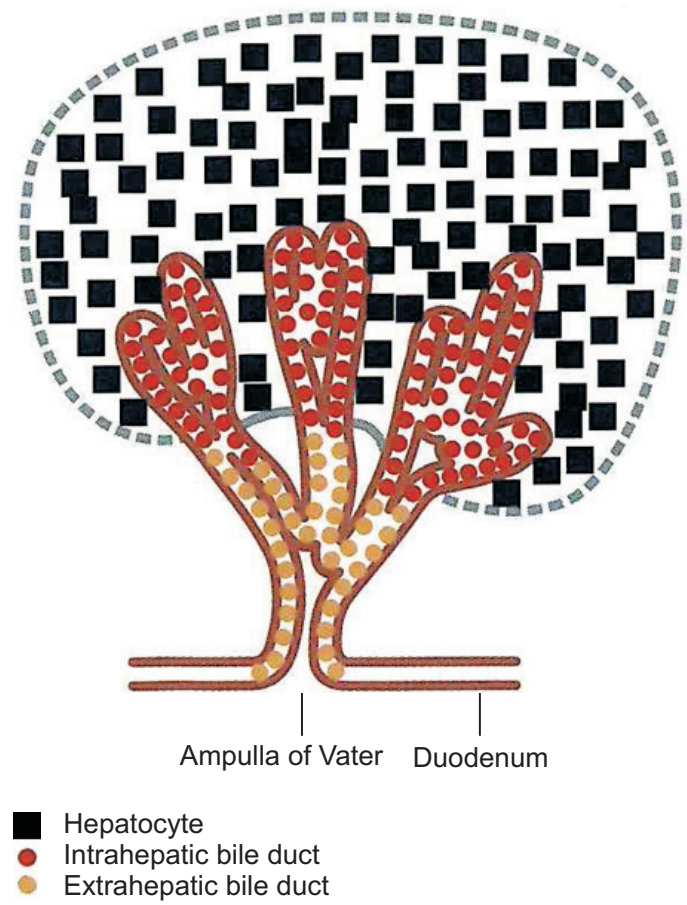

Fig. 11. The development of the intrahepatic bile duct, extrahepatic bile duct and hepatocytes differs in mice (18.5 embryonic days). The hepatocytes are directly derived from albumin-positive hepatoblasts in the hepatic diverticulum, while the intrahepatic bile ducts are derived via the ductal plate from albumin-positive hepatoblasts in the hepatic diverticulum, and the extrahepatic bile duct is derived from albumin-negative hepatoblasts in the hepatic diverticulum. Adapted with permission from Nishikawa Y and Enomoto . $^{40}$ 
four subtypes of IPNB are not the same as those of IPMN. ${ }^{35} \mathrm{We}$ therefore reviewed the histology of a total of 69 cases of IPNB with an emphasis on the four subtypes in consideration of the two abovementioned categories (types 1 and 2). The number of cases of individual subtypes and the proportions of types 1 and 2 in these subtypes are shown in Table 5.

\section{Intestinal IPNB}

This subtype was found in 33 cases (47.8\%) of IPNBs. Intestinal IPNB (iIPNB) is defined by neoplastic epithelia lining the fibrovascular cores, showing columnar cells with pseudostratified, cigar-shaped nuclei and basophilic or amphophilic cytoplasm with variable amounts of supranuclear mucin, resembling colorectal mucosal epithelia and their derived neoplasms. ${ }^{1,6}$ Goblet-like cells with micropapillary features are variably admixed. The epithelial cells in iIPNB usually have intermediateor high-grade dysplasia. Immunohistochemically, CK20 and/ or CDX2 (>50\% of whole tumor) are constantly expressed in their cytoplasm. iIPNB with typical histology is almost always positive for CDX2. Rare iIPNB cases that are negative or only focally positive for CDX2 are diffusely positive for CK20. MUC2 is usually detectable in goblet cells in IIPNB.

Interestingly, some iIPNBs present mainly villous structures, and the overall picture is highly reminiscent of colorectal villous neoplasms; thus, they resemble intestinal IPMNs showing villous patterns (Fig. 3). Other iIPNBs resemble papillovillous or papillotubular colorectal neoplasms (Fig. 4). The villous neoplasms may deserve to be classified as type 1 based on its similarities to intestinal IPMN and is usually detected in the intrahepatic bile duct (Nakanuma et al., unpublished observation). Some cases of papilla-tubular or papilla-villous neoplasms additionally present with cribriform or solid structures and crowded tubular patterns as well as more aggressive histopathological features and can be considered to be type 2-these are usually detected in the extrahepatic bile ducts. Type 1 iIPNBs frequently show gene mutations, such as GNAS and KRAS mutations, as seen in intestinal IPMN, while type 2 gene mutations include SMAD4, PIK3CA, $A P C$ and CTNNB1, which are seen in colorectal neoplasms (Nakanuma et al., unpublished observation).

\section{Gastric IPNB}

This subtype was found in 17 cases (23.2\%) of IPNBs. The epithelium lining gastric IPNB (gIPNB) is composed of tall columnar cells with basally oriented nuclei and abundant pale mucinous cytoplasm, reminiscent of gastric foveolar epithelium. Pyloric glands are frequently intermingled with these foveolar patterns. Scattered goblet cells can be seen. gIPNBs are moderately to extensively positive for MUC5AC in the foveolar areas and MUC6 can be detected in the pyloric gland portions. Generally, gIPNBs with innocuous epithelia corresponding to low-or intermediate grade dysplasia, particularly those in the intrahepatic bile duct, resemble gastric IPMNs and thus deserve to be classified as type 1 (Fig. 5). Interestingly, low papillary or flat IPMNs of the gastric type with cystic changes characteristically involving the branch pancreatic ducts are not found in gIPNB.

Other IPNBs composed of columnar epithelia with high-grade dysplasia and variably expressing MUC5AC and MUC6 show more complicated structures, such as irregular papillary or tubular or microcystic changes with atypical features, frequent highgrade dysplasia and even overt malignant changes; however, a foveolar appearance is consistently observed, and pyloric glands features with cuboidal or low columnar epithelial cells are recognizable (Fig. 6). They may belong to the gastric type and therefore deserve to be categorized as type 2 gIPNB. They are generally seen in the extrahepatic bile ducts but can also occur in the intrahepatic bile ducts.

\section{Pancreatobiliary IPNB}

IPNBs showing ramifying fine and thin branches and papillae covered by cuboidal to low columnar epithelia with round, hyperchromatic nuclei, prominent nucleoli, variably acidophilic or amphophilic or pale cytoplasm, and a less mucinous appearance are recognized as PB type. Pancreatobiliary IPNB (pbIPNB) was found in 11 cases in the present study (15.9\%) of IPNBs. These lesions are usually immunohistochemically positive for S100P and MUC1. MUC5AC is frequently negative in these neoplastic cells. Some pbIPNBs showing more regular and monotonous structures with a thin fibrovascular stalk covered by low columnar or cuboidal biliary epithelia are regarded as type 1 (Fig. 7) and also seem similar to the prototypical PB type of IPMN. The structures of other IPNBs present with irregular papillary architecture with more stratified nuclei and solid or comedo-like structures with atypical structures and cells and nuclei including overt malignant changes; these are classified as type 2 (Fig. 8).

\section{Oncocytic IPNB}

This subtype was found in eight cases (11.6\%) of IPNBs and usually has complex and arborizing papillae with delicate fi-

Table 5. The Numbers of the Four Subtypes and the Incidence of Types 1 and 2 among the Four Subtypes of Intraductal Papillary Neoplasm of the Bile Duct

\begin{tabular}{lccccc}
\hline & \multirow{2}{*}{ Total } & \multicolumn{3}{c}{ Subtype } \\
\cline { 3 - 6 } & & Intestinal & Gastric & Pancreatobiliary & Oncocytic \\
\hline Total & 69 & 33 & 17 & 11 & 8 \\
Proportion of type 1:2 & $19: 50$ & $5: 28$ & $7: 10$ & $4: 7$ & $3: 5$ \\
\hline
\end{tabular}


brotic and edematous stroma. The papillae are lined by one to several stratified layers of cuboidal to columnar cells with abundant eosinophilic granular cytoplasm and occasional hyaline globules with high-grade dysplasia. The nuclei are hyperchromatic, round, large and fairly uniform and typically contain a single and prominent nucleolus. Some hyperchromatic nuclei are rather pyknotic. Goblet cells may be interspersed among the oncocytic cells. The neoplastic cells often form intraepithelial lumina. In some cases, these intraepithelial lumina produce a cribriform pattern, and in others, the epithelium of the adjacent papillae seems to fuse, producing a solid growth pattern punctuated by small vessels.

Some oncocytic IPNBs (oIPNBs) show a more complex and solid structure or papillary architecture with one- to two-layered columnar cells with variably oncocytic cytoplasm; others show fine fibrovascular branches lined by stratified oncocytic columnar or cuboidal neoplastic epithelia with secondary lumina; the latter deserve to be classified as type 1 oIPNBs (Fig. 9) and resemble a prototypical oncocytic IPMNs of the pancreas, whereas the former show a complex and solid growth pattern and can be regarded as type 2 (Fig. 10).

Taken together, these findings suggest that some IPNBs present with subtypes similar to the four subtypes of IPMN, while the histology of others differs to varying degrees from the four prototypical IPMN subtypes. Interestingly, type 1 IPNB is frequently found in the intrahepatic bile duct, while type 2 IPNB is found in the extrahepatic bile duct, suggesting that the anatomical site of the biliary tree where IPNBs arise may also influence in the development of the two categories of IPNB subtypes.

\section{TREATMENT}

Because of possible recurrent biliary obstruction in all cases and frequent high-grade dysplasia with minimal invasion, all patients with IPNB should be treated. ${ }^{18}$ All patients without distant metastasis are surgical candidates and should be resected in a manner similar to surgery for cholangiocarcinoma. ${ }^{18,28}$ If superficial spreading is extensive with a positive resection margin, resection of the extensive biliary tree and pancreaticoduodenectomy may be performed. ${ }^{41}$

\section{MIMICKERS OF IPNB}

Several biliary tumors other than IPNB are also present or are associated with intraductal growth in the bile ducts. Typically, conventional cholangiocarcinomas (CCAs) present nodular/ sclerosing growth of the affected bile ducts with luminal stenosis or obliteration, and the luminal surface of the affected and adjacent bile duct mucosa are grossly granular or coarse. These conventional, nodular/sclerosing CCAs occasionally present with intraductal growth showing invasive carcinoma with desmoplasia continuous with invasive ductal and periductal inva- sive carcinoma. Metastatic carcinomas, particularly those from colorectal adenocarcinoma, also show grossly visible intraluminal growth in the bile duct. ${ }^{23}$

Several examples of disease entities that mimic IPNB are described below.

\section{Conventional CCAs with intraductal growth versus IPNB associated with an invasive carcinoma}

IPNB is not difficult to diagnose when stromal invasion develops in the basal sites (IPNB associated with an invasive carcinoma). However, when IPNB shows invasion of adenocarcinoma in the fibrovascular stroma with a desmoplastic reaction, such invasion results in the fibrous widening of fibrovascular cores and deformity of the papillary structures forming nodularities, making it difficult to differentiate from some conventional CCAs with intraluminar nodular growth. Micropapillary components corresponding to biliary intraepithelial neoplasms (BilINs) preceding invasive CCAs have occasionally been seen on their luminal surface or the adjoining mucosa of these invasive conventional CCAs. ${ }^{4,14}$ The clinical and pathologic differentiation of these nodular sclerosing CCAs from IPNB associated with an invasive carcinoma are occasionally controversial. At present, "papillary components comprising $>50 \%$ of the intraductal tumor and papillary growth typically $>5 \mathrm{~mm}$ in height" are the proposed diagnostic criteria for IPNB, particularly type 2 IPNB associated with an invasive carcinoma; ${ }^{22}$ these criteria will be useful for differentiation from conventional CCA with intraluminal nodular growth.

In addition, in some cases of IPNB, invasion predominantly develops at the lateral-spreading flat or non-papillary neoplastic areas immediately around as well as remote from the main tumor of the IPNB, and this invasive carcinoma is occasionally present as nodular sclerosing CCA. ${ }^{24}$ Similar phenomena have also been reported in IPMN cases in which carcinoma develops in the non-papillary flat neoplastic area around the IPMN. ${ }^{7}$

\section{Biliary intraepithelial neoplasms}

BilINs are another type of preinvasive lesion of CCA. They are flat or non-papillary. In most cases, conventional nodular/ sclerosing CCAs follow a BilIN. They are graded as BilIN-1 and BillN-2 (low/intermediate dysplasia) and BilIN-3 (high-grade dysplasia, carcinoma in situ). In fact, BilINs that appear similar to intraepithelial neoplasia are frequently encountered in the mucosa surrounding a conventional CCA. Interestingly, some BilINs present with pseudopapillary- or micropapillary-like lesions, but their heights are less than $3 \mathrm{~mm}$ (micropapillary BilINs), which differentiates them from IPNB. ${ }^{24}$

\section{Intraductal tubulopapillary neoplasms of the bile duct}

The most important microscopic characteristics of intraductal tubulopapillary neoplasms (ITPNs) are as follows: $:^{25,26}$ (1) the tumor is mainly composed of tubular configurations with abor- 
tive papillary components, and the tumor cells contain no cytoplasmic mucin; (2) uniform high-grade dysplasia is observed throughout the tumor, and no low-grade areas are seen. These features contrast with those of IPNBs. In IPNBs, the tumor cells frequently show the apparent production of intercellular and extracellular mucin. In addition, the transition from low- to high-grade areas can be seen in IPNBs. The histological subtyping used for IPNBs is not applicable to ITPNs. This may also be a biliary counterpart of pancreatic ITPN.

\section{Pyloric gland adenoma and others}

Pyloric gland adenoma, which resembles a pyloric gland and expresses MUC6, has been reported in the bile duct. ${ }^{31}$ In addition, we have experienced grossly visible intraluminal tumors showing a tubular neoplasm without invasion into the ductal wall and periductal tissues. Some of these cases showed intestinal differentiation and could be described as tubular colorectal neoplasms. These tubular or glandular neoplasms will be characterized in the future.

\section{CONCLUSIONS}

The gross and microscopic features and biological behaviors of IPNBs are highly variable, suggesting that IPNB could be a complex biliary disease. Some IPNBs share many features with pancreatic IPMN (type 1), while other cases display various differences from IPMN and also present with cribriform or solid or tubular components (type 2). Interestingly, the four subtypes of IPNB were also dividable into categories either reminiscent of the four prototypical subtypes of IPMN (type 1) or variably different from IPMN (type 2). Type 1 IPNBs were usually found in the intrahepatic bile ducts, while type 2 IPNBs were predominantly found in the extrahepatic bile ducts, suggesting that anatomical factors influence the development of these two categories. The recognition of these two categories of IPNB with reference to IPMN and its anatomic location along the biliary tree improves our understanding of the variable pathologies, clinical behaviors and manifestations of IPNB.

\section{CONFLICTS OF INTEREST}

No potential conflict of interest relevant to this article was reported.

\section{REFERENCES}

1. Nakanuma Y, Curado M-P, Franceschi S, et al. Intrahepatic cholangiocarcinoma. In: Bosman FT, Carneiro F, Hruban RH, Theise ND, eds. WHO of tumours of the digestive system. Lyon: International Agency for Research on Cancer, 2010:217-224.

2. Albores-Saavedra J, Adsay NV, Crawford JM, et al. Carcinoma of the gallbladder and extrahepatic bile ducts. In: Bosman FT,
Carneiro F, Hruban RH, Theise ND, eds. WHO classification of tumours of the digestive system. Lyon: International Agency for Research on Cancer, 2010:266-273.

3. Chen TC, Nakanuma Y, Zen Y, et al. Intraductal papillary neoplasia of the liver associated with hepatolithiasis. Hepatology 2001;34(4 Pt 1):651-658.

4. Albores-Saavedra J, Murakata L, Krueger JE, Henson DE. Noninvasive and minimally invasive papillary carcinomas of the extrahepatic bile ducts. Cancer 2000;89:508-515.

5. Nakanuma Y, Kakuda Y, Uesaka K, et al. Characterization of intraductal papillary neoplasm of bile duct with respect to histopathologic similarities to pancreatic intraductal papillary mucinous neoplasm. Hum Pathol 2016;51:103-113.

6. Zen Y, Fujii T, Itatsu K, et al. Biliary papillary tumors share pathological features with intraductal papillary mucinous neoplasm of the pancreas. Hepatology 2006;44:1333-1343.

7. Adsay NV, Kloeppel G, Fukushima N, et al. Intraductal neoplasms of the pancreas. In: Bosman FT, Carneiro F, Hruban RH, Theise ND, eds. WHO classification of tumours of the digestive system. Lyon: International Agency for Research on Cancer, 2010:304313.

8. Zen Y, Fujii T, Itatsu K, et al. Biliary cystic tumors with bile duct communication: a cystic variant of intraductal papillary neoplasm of the bile duct. Mod Pathol 2006;19:1243-1254.

9. Zen Y, Sasaki M, Fujii T, et al. Different expression patterns of mucin core proteins and cytokeratins during intrahepatic cholangiocarcinogenesis from biliary intraepithelial neoplasia and intraductal papillary neoplasm of the bile duct: an immunohistochemical study of 110 cases of hepatolithiasis. J Hepatol 2006;44:350358.

10. Hoang MP, Murakata LA, Katabi N, Henson DE, Albores-Saavedra J. Invasive papillary carcinomas of the extrahepatic bile ducts: a clinicopathologic and immunohistochemical study of 13 cases. Mod Pathol 2002;15:1251-1258.

11. Jung G, Park KM, Lee SS, Yu E, Hong SM, Kim J. Long-term clinical outcome of the surgically resected intraductal papillary neoplasm of the bile duct. J Hepatol 2012;57:787-793.

12. Rocha FG, Lee H, Katabi N, et al. Intraductal papillary neoplasm of the bile duct: a biliary equivalent to intraductal papillary mucinous neoplasm of the pancreas? Hepatology 2012;56:1352-1360.

13. Yeh TS, Tseng JH, Chen TC, et al. Characterization of intrahepatic cholangiocarcinoma of the intraductal growth-type and its precursor lesions. Hepatology 2005;42:657-664.

14. Ying S, Ying M, Liang W, et al. Morphological classification of intraductal papillary neoplasm of the bile duct. Eur Radiol 2018;28:1568-1578.

15. Fukumura Y, Nakanuma Y, Kakuda Y, Takase M, Yao T. Clinicopathological features of intraductal papillary neoplasms of the bile duct: a comparison with intraductal papillary mucinous neoplasm of the pancreas with reference to subtypes. Virchows Arch 2017;471:65-76.

16. Nakanuma Y, Uesaka K, Miyayama S, Yamaguchi H, Ohtsuka M. 
Intraductal neoplasms of the bile duct. A new challenge to biliary tract tumor pathology. Histol Histopathol 2017;32:1001-1015.

17. Ohtsuka M, Kimura F, Shimizu H, et al. Similarities and differences between intraductal papillary tumors of the bile duct with and without macroscopically visible mucin secretion. Am J Surg Pathol 2011;35:512-521.

18. Park HJ, Kim SY, Kim HJ, et al. Intraductal papillary neoplasm of the bile duct: clinical, imaging, and pathologic features. AJR Am J Roentgenol 2018;211:67-75.

19. Minagawa N, Sato N, Mori Y, Tamura T, Higure A, Yamaguchi $\mathrm{K}$. A comparison between intraductal papillary neoplasms of the biliary tract (BT-IPMNs) and intraductal papillary mucinous neoplasms of the pancreas (P-IPMNs) reveals distinct clinical manifestations and outcomes. Eur J Surg Oncol 2013;39:554-558.

20. Tsai JH, Yuan RH, Chen YL, Liau JY, Jeng YM. GNAS Is frequently mutated in a specific subgroup of intraductal papillary neoplasms of the bile duct. Am J Surg Pathol 2013;37:1862-1870.

21. Fujikura K, Fukumoto T, Ajiki T, et al. Comparative clinicopathological study of biliary intraductal papillary neoplasms and papillary cholangiocarcinomas. Histopathology 2016;69:950-961.

22. Nakanuma Y, Jang KT, Fukushima N, et al. A statement by the Japan-Korea expert pathologists for future clinicopathological and molecular analyses toward consensus building of intraductal papillary neoplasm of the bile duct through several opinions at the present stage. J Hepatobiliary Pancreat Sci 2018;25:181-187.

23. Yamao T, Hayashi H, Higashi T, et al. Colon cancer metastasis mimicking intraductal papillary neoplasm of the extra-hepatic bile duct. Int J Surg Case Rep 2015;10:91-93.

24. Nakanuma Y, Uchida T, Sato Y, Uesaka K. An S100P-positive biliary epithelial field is a preinvasive intraepithelial neoplasm in nodular-sclerosing cholangiocarcinoma. Hum Pathol 2017;60:4657.

25. Schlitter AM, Jang KT, Klöppel G, et al. Intraductal tubulopapillary neoplasms of the bile ducts: clinicopathologic, immunohistochemical, and molecular analysis of 20 cases. Mod Pathol 2015;28:1249-1264.

26. Yamaguchi H, Shimizu M, Ban S, et al. Intraductal tubulopapillary neoplasms of the pancreas distinct from pancreatic intraepithelial neoplasia and intraductal papillary mucinous neoplasms. Am J Surg Pathol 2009;33:1164-1172.

27. Nakanuma Y, Sudo Y. Biliary tumors with pancreatic counterparts. Semin Diagn Pathol 2017;34:167-175.

28. Ohtsuka M, Shimizu H, Kato A, et al. Intraductal papillary neo- plasms of the bile duct. Int J Hepatol 2014;2014:459091.

29. Schlitter AM, Born D, Bettstetter M, et al. Intraductal papillary neoplasms of the bile duct: stepwise progression to carcinoma involves common molecular pathways. Mod Pathol 2014;27:73-86.

30. Nakanuma Y, Miyata T, Uchida T, Uesaka K. Intraductal papillary neoplasm of bile duct is associated with a unique intraepithelial spreading pattern. Int J Clin Exp Pathol 2016;9:11129-11138.

31. Hatzibougias DI, Fouzas I, Bobos M, et al. Tubular pyloric gland adenoma of the left and right hepatic ducts: report of a unique case with immunohistochemical and molecular studies. Int J Surg Pathol 2016;24:347-352.

32. Sumazaki R, Shiojiri N, Isoyama S, et al. Conversion of biliary system to pancreatic tissue in Hes1-deficient mice. Nat Genet 2004;36:83-87.

33. Shiojiri N. Development and differentiation of bile ducts in the mammalian liver. Microsc Res Tech 1997;39:328-335.

34. Spence JR, Lange AW, Lin SC, et al. Sox17 regulates organ lineage segregation of ventral foregut progenitor cells. Dev Cell 2009;17:62-74.

35. Carpino G, Renzi A, Franchitto A, et al. Stem/progenitor cell niches involved in hepatic and biliary regeneration. Stem Cells Int 2016;2016:3658013.

36. Wang Y, Lanzoni G, Carpino G, et al. Biliary tree stem cells, precursors to pancreatic committed progenitors: evidence for possible life-long pancreatic organogenesis. Stem Cells 2013;31:19661979.

37. Miyata T, Uesaka K, Nakanuma Y. Cystic and papillary neoplasm at the hepatic hilum possibly originating in the peribiliary glands. Case Rep Pathol 2016;2016:9130754.

38. Uchida T, Yamamoto Y, Ito T, et al. Cystic micropapillary neoplasm of peribiliary glands with concomitant perihilar cholangiocarcinoma. World J Gastroenterol 2016;22:2391-2397.

39. Nakanuma Y. A novel approach to biliary tract pathology based on similarities to pancreatic counterparts: is the biliary tract an incomplete pancreas? Pathol Int 2010;60:419-429.

40. Nishikawa Y, Enomoto K. Anatomy of junctions of intrahepatic bile ducts, extrahepatic bile ducts and intestine. In: Nakanuma Y, ed. Textbook of of biliary tract. Tokyo: Nanzando Publishing, 2015:14-19.

41. Ohtsuka M, Kimura F, Shimizu H, et al. Surgical strategy for mucin-producing bile duct tumor. J Hepatobiliary Pancreat Sci 2010;17:236-240. 